HLGHES, H. \& ADAIIS, H. I. Temination of shock as a secondary reinforcer The eftect of replications. Psychonomic Science. 1967. 8. $178-180$.

LOLORDO, V. M. Positive conditioned reinforcement from aversive situations. Psychological Bulletin, 1969. 72. 193-203.

SIEGEL, P. S., \& MILBY, J. B.. JR. Secondary reinforcement in relationship to shock termination: Second chapter. Psychological
Bulletin. 1969.72,146-156.

YAC,IA. li. \& ALLEN. H. I. The development of a conditioned positive reinforcer based upon the termination of shock. Psychonomic Science, 1964. I. $363-364$.

ZIMUERUA rats based on secondary reinforcement. Journal of Comparative \& Phrsiological Psychology, 1959, 52, 35-42.

\section{Double-intermittent reward scheduling and secondary-reinforcer strength: Discriminated escape*}

\author{
D. GENE DAVENPORT and A. JOHN ESCHENBRENNER $†$ \\ St. Louis University, St. Louis, Mo. 63103
}

A discriminated escape procedure was employed to train two groups of rats to activate a nose press in the presence of a tone to escape a $1 \cdot \mathrm{mA}$ shock. Escape was allowed on a continuous or an intermittent schedule. Each group was then divided into three subgroups, two of which were required to learn a leverpress response where the only reward was the tone, presented on a continuous or an intermittent reinforcement schedule. The third subgroup served as a no-tone control. A durable secondary reinforcer was established which was a function of the secondary reinforcement schedule during testing.

Recent reviews (LoLordo, 1969; Siegel \& Milby, 1969) conclude that the existence of a durable and effective secondary reinforcer in aversive drive situations was still largely a matter of conjecture. They felt that no completely adequate demonstration of the phenomenon in question had, as of that time, been tendered. However, recent results from our own laboratory, not included in these reviews and apparently not suffering from the problems of early studies (Davenport \& Lerner, 1968; Davenport, 1970), have indicated that a neutral stimulus paired with escape from an aversive situation can acquire reinforcing properties when it functions as a discriminative stimulus for escape. It was felt, as a consequence, that current research should concentrate on the investigation of parameters that influence the establishment and strength of secondary reinforcers in situations in which the motivation for behavior is aversive. Schedule of reinforcement, one of the parameters that affects the establishment and strength of secondary reinforcers in

*This experiment is based on a thesis submitted by the second author under the guidance of the first author to the Graduate School, St. Louis University, in partial fulfillment of requirements for the MS degree.

+ Now at McDonnell-Douglas Corporation, St. Louis, Mo. 63042 . appetitive drive situations, furnished the necessary point of departure. The early work of Zimmerman (1959) provided some rudimentary evidence for the efficacy of a two-phase or double-intermittent schedule of reinforcement. Fox \& King (1961), in an effort to avoid the criticisms leveled at Zimmerman, added some appropriate controls and found evidence for the superiority of the double-intermittent procedure. Davenport \& Sardello (1966), in a partial replication of Fox \& King, introduced other methodological changes to insure mobility of the Ss and minimize secure additional evidence for the superiority of the double-intermittent schedule over procedures involving continuous reinforcement.

The present experiment was designed to seek a verification of the superiority of employing a double-intermittent schedule, as opposed to a single-phase intermittent schedule, in establishing a stable and effective positive secondary reinforcer in an aversive-drive situation involving discriminated escape.

\section{METHOD}

The Ss were 36 naive male albino rats of the Sprague-Dawley strain, approximately 80 days old on the first day of the experiment.

The apparatus consisted of two standard stimulus redundancy. They were able to single-lever Gerbrands-Skinner boxes in sound-attenuating chambers. Each box was equipped with a Lehigh Valley pigeon key, used to record nose presses, mounted on the back wall of the box, $10 \mathrm{~cm}$ from the lever and $5 \mathrm{~cm}$ above the grid floor. All programming and recording was automatically controlled, and escape from a 1-mA shock, provided by a Lehigh Valley constant-current scrambled shocker, was used as a reinforcer during the training period.

A procedure quite similar to Davenport $\&$ 'Sardello (1966) was used. Each S was given two 60-min training sessions in which every nose press resulted in a $60-\mathrm{sec}$ escape from shock and the termination of two stimulus lights. All Ss were then placed on a schedule in which a tone was presented on a VI 4-sec schedule, and a nose press in the presence of the tone resulted in a 30-sec escape from shock and termination of the tone. All responses in the absence of the tone produced an additional $10-\mathrm{sec}$ delay in the tone presentation while the shock continued. Each $\mathrm{S}$ was given three 60-min training sessions under these conditions. The variable interval presentation was subsequently extended to a VI $15-\mathrm{sec}$ schedule subject to the above $10-\mathrm{sec}$ nonresponse restriction for a total of 2 more hours before partial reinforcement training began. The $18 \mathrm{Ss}$ receiving partial reinforcement continued to receive 50 tones on a VI $15 \cdot \mathrm{sec}$ schedule throughout the remaining training sessions. However, the proportion of nose-press responses made in the presence of the tone resulting in reward was decreased to $60 \%$ for $2 \mathrm{~h}$ of training, $40 \%$ for $2 \mathrm{~h}$, and $10 \%$ for $3 \mathrm{~h}$, with the number of reinforcements given reducing from 50 to 5 . The $18 \mathrm{Ss}$ on continuous reinforcement were placed on a VI $45-\mathrm{sec}$ schedule for $2 \mathrm{~h}$, a VI $83-\mathrm{sec}$ schedule for $2 \mathrm{~h}$, and finally, a VI 420 -sec schedule for tone presentations for $3 \mathrm{~h}$. This latter procedure had the effect of presenting the tones at the same rate as those actually reinforced in the partial reinforcement group so that equal reinforcements over equal training times was maintained. There was no more reliable cue than the tone indicating when escape was possible in this discriminated escape procedure.

For testing the strength of the tone as a secondary reinforcer, a lever was added, and each response resulted in a tone on a continuous schedule for one-third of the group receiving partial pairing during training and a tone on a VR-4 schedule for another third. The other third of the groups received no tones during testing. A nose press in the presence of the tone terminated the tone, but not the shock. The group receiving continuous pairing of 


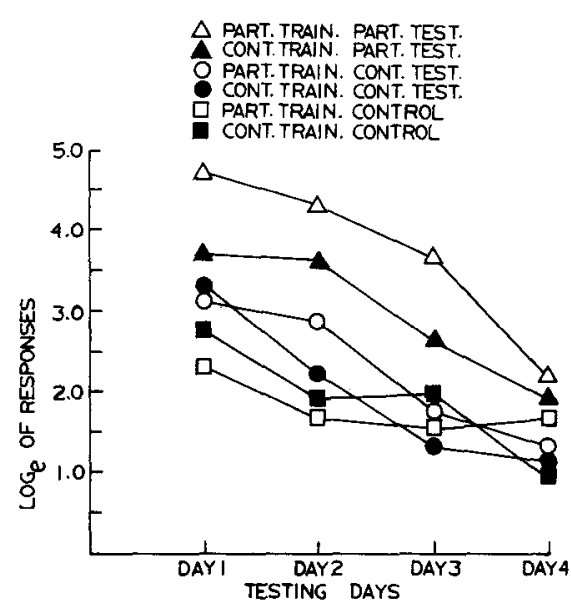

Fig. 1. Effects of continuous and partial reinforcement during training and testing for each session.

tone and escape from shock during training was similarly divided and tested. The number of leverpresses and the latency of responding to the tone over a 30-min session on each of 4 successive days were the response measures recorded.

\section{RESULTS AND DISCUSSION}

An analysis of variance was performed on the reciprocal of the response latencies to the tone on the last day of training, using the median latency to represent a $S$ 's performance. There were no significant differences among the six groups $[F(5,30)=.15, p>.25]$, indicating that partial reinforcement during training did not interfere with the continuation of the tone as a discriminative stimulus and that randomization was effective. It should be noted, however, that discrimination was relatively poor for all groups, the average median latency ranging from $9.81 \mathrm{sec}$ to $12.32 \mathrm{sec}$ for the various groups just prior to testing.

The measure of the effectiveness of the tone as a secondary reinforcer was the number of lever responses produced when the only reinforcement was the tone. The response totals for each $S$ for each of the four sessions were transformed to logarithms. Figure 1 provides the mean $\log _{\mathbf{e}}$ responses for each group during testing. A between-groups, within-session analysis of variance on the number of responses during testing resulted in significant effects due to testing reinforcement schedule $[F(2,30)=17.27$, $\mathrm{p}<.001]$, to testing sessions $[\mathrm{F}(3,90)=40.49, \mathrm{p}<.001]$, and to the Testing by Sessions interaction $[F(6,90)=3.35, p<.01]$. No other effects were significant. Thus, the overall performance of the $S$ s receiving intermittent reinforcement by the tone during testing was superior to that of the controls, with the continuous reinforcement Ss falling between. Also, the number of responses per session made by all groups decreased as the testing was repeated; and the size of the superiority of the partial over continuous. and continuous over controls was greater on the early sessions than on the last ones. As an additional analysis, a Duncan multiple-range test of the total performance indicated that the partial-training/partial-testing group differed significantly from all the other groups except continuous training/partial testing.

Taken together with the previous analysis, it was concluded that if partial primary reinforcement during training had an effect at all, it was in connection with the intermittent presentation of the tone, for only this combination produced a significantly larger response output than both control groups. It would appear that both intermittent and continuous primary reinforcement during training resulted in the establishment of the tone as a secondary reinforcer when contrasted with controls. However, the effect was quite transient when the tone was presented for each response, for the continuous testing Ss performed no better than controls by the third test session (Fig. 1).

A similar analysis of variance was performed on the reciprocal nose-response latency to the first eight lever-produced tone occurrences during testing (one S only produced eight tones). The only significant effect was due to trials $[F(7,140)=3.16$, $p<.01]$, indicating that the speed of the response was slowing for all groups with repeated tone presentation. However, the rank order for the four groups on mean reciprocal latency over eight trials was the same as their rank order on secondary reinforcement. If the training were completely successful, it would be expected that response latencies for the Ss trained with the intermittent schedule would have continued to be short for more than eight trials. As indicated earlier, the discriminative control over responding during training was not all that could be desired, and improved training procedures might well enhance the superiority of partial training over continuous training not statistically reliable in the present results.

In general, the data from the present study are similar to those reported by Fox \& King (1961) and Davenport \& Sardello (1966) for a secondary reinforcement effect based on schedules of positive reinforcement. In all three studies the double-intermittent schedule ranked first, followed by the two single-intermittent schedules, and finally, the continuous-reinforcement schedules, with controls ranking last when available. In Davenport \& Sardello the primary-reinforcement schedule during training was the significant factor, and in the present study, the secondary-rcintorcement schedule during testing was. In these studies, as well as in that of Fox \& King (1961), both phases seemed to have an influence in terms of the direction of the effect favoring intermittent schedules, but the differences were not always statistically reliable. The presence of these somewhat transient effects, occurring sometimes in one place, at other times in another, would appear to indicate the presence of true effects, the problem being to identify the actual condition that produces them.

Another consistency between the results of Davenport \& Sardello and the present study should be stressed. In both studies it was found that the effectiveness of the tone as a secondary reinforcer in sustaining the lever response had a perfect rank-order correlation with the tone's discriminative control over the nose press, this in spite of rather poor discrimination training in the present study. These results would seem to be consistent with the discriminative-stimulus hypothesis of secondary reinforcement (e.g., that a stimulus maintained as a cue for one response will function as a reinforcer for another response). This interpretation seems reasonable, even though the statistical evidence in an individual study is not strong.

In conclusion, it would seem clear that a cue in discriminated escape can function as a secondary reinforcer for the acquisition of a new response. Although the two-phase intermittent reinforcement schedule has again produced the greatest amount of responding, this time in discriminated escape, only the schedule involving the presentation of the tone could be statistically demonstrated as influencing responding.

\section{REFERENCES}

DAVENPORT, D. G. The secondary positive reinforcing properties of the cue in "automated" discriminated escape. Psychonomic Science, 1970, 21, 149-151.

DAVENPORT, D. G., \& LERNER, J. J. The cue in discriminated escape conditioning as a secondary positive reinforcer. Psychonomic Science, 1968, 13, 47-48.

DAVENPORT, D. G., \& SARDELLO, R. J. Double intermittent reward scheduling and secondary reinforcer strength. Psychonomic Science, $1966,6,417-418$.

FOX, R. E., \& KING, R. A. The effect of reinforcement scheduling on the strength of a secondary reinforcer. Journal of Comparative \& Physiological Psychology, 1961, 54, 266-269.

LoLORDO, V. M. Positive conditioned reinforcement from aversive situations. Psychological Bulletin, 1969, 72 193-203. 
SIEGEL, P. S., \& MILBY, J. B., JR. Secondary reinforcement in relation to shock termination: Second chapter. Psychological Bulletin, 1969, 72, 146-156.
ZIMMERMAN, D. W. Durable performance in rats based on secondary reinforcement. Journal of Comparative \& Physiological Psychology, 1959, 52, 35.42.

\section{The effect of disconfirming an expectancy of time-out from shock in the double runway*}

\author{
JOSEPH V. LAMBERT $\dagger$ \\ Temple University, Philadelphia, Pa. 19122
}

In a double-runway apparatus using shock escape as the reinforcement, Lambert \& Hammond (in press) showed that the Amselian frustration effect (FE) manifests itself as a marked slowing down in the second runway, when an expected relief from shock is not forthcoming. The present experiment was performed to determine if an animal builds up a particular expectancy of timeout from shock and what the effect might be of disconfirming this expectancy by giving a substantially shorter period of shock relief. The "reversed FE" again appeared when a particular expectancy of timeout from shock was disconfirmed. Also, Runway 1 speeds were seen to decrease when this frustration treatment was introduced.

In an attempt to extend Amselian frustration theory from the appetitive to the aversive realm, Lambert \& Hammond (in press) performed an experiment in

*This research was supported in part by NIMH Grant $16540-02$ and by a grant-in-aid from Temple University to L. J. Hammond.

$\dagger$ The author wishes to thank $W$. Love for his aid in running the animals and L. J. Hammond and P.J. Bersh for their helpful criticisms of the manuscript. Reprints may be obtained from Joseph V. Lambert, Department of Psychology, Temple University, Philadelphia, Pa. 19122.
$2 \mathrm{sec}$ of being confined under shock prior to the raising of the $G 1$ exit door to $R 2$. The response of running down $R 2$ to $G 2$ ended in relief from shock on all trials. This latter procedure was considered the frustration condition. It was found in this study that animals on frustrative nonrelief trials ran significantly slower in R2 than they did on relief trials. In a second experiment this "reversed FE" was not evident until approximately 20 trials had occurred, when running to $G 1$ was partially reinforced from the beginning. Lambert \& Hammond (in press) argued that this latter finding showed that the slowing down was not simply due to differing nonassociative factors occurring on the two types of trials. That is, they suggested that the "reversed FE" depended on associative factors (expectancy of reinforcement in G1 which could only occur after a number of trials had taken place), as was true in the appetitive case (Roussel, 1952; Amsel \& Hancock, 1957; Wagner, 1959). A possible criticism of this argument is that the effect may be due to the interruption of shock on relief trials but not on frustration trials. The present experiment was performed to take into account this criticism of the difference in shock continuities. Here, animals were trained to expect a certain period of relief from shock for the response of running to $\mathrm{G} 1$, whereupon they were frustrated in that their expectancy of a particular duration. of shock offset was disconfirmed. However, this procedure interrupted the shock and afforded the animals a minimum timeout from shock.

\section{SUBJECTS}

Sixteen male albino rats of the Sprague-Dawley strain, weighing $150-175 \mathrm{~g}$, served as Ss. They were housed individually and given ad lib food and water.

\section{APPARATUS}

A straight alley similar to that used in Amsel \& Roussel's 1952 study was used, but with the addition of an electrifiable floor. The alley was $10 \mathrm{ft}$ long and consisted consecutively of a $1-\mathrm{ft}$ startbox (SB), a 3-ft first runway (R1), a 1-ft first goalbox (G1), a 4-ft second runway (R2), and finally, a 1-ft second goalbox (G2). Guillotine doors separated each of these compartments. The inside dimensions were 4 in. high $\times 2-5 / 8$ in. wide. The hinged top was of clear Plexiglas. The shocking surface was made of two L-shaped aluminum runners, each of which formed one wall and one half of the floor. A 5/8-in. gap ran down the middle of the runway and separated the floor portion of the runners. Sigma photocells were placed below this gap in the floor, one 3 in. outside of SB 